\title{
The Role of Muscle Relaxants - Spasmolytic (Thiocochlicoside) in Postoperative Pain Management after Mastectomy and Breast Reconstruction
}

\author{
Maria Bourazani ${ }^{1 *}$, Effie Papageorgiou ${ }^{2}$, Georgios Zarkadas ${ }^{3}$, Theodora \\ Petrakopoulou $^{3}$, Evridiki $\mathrm{Kaba}^{4}$, Georgia Fasoi ${ }^{4}$, Martha Kelesi ${ }^{5}$
}

\begin{abstract}
Purpose: Post-operative pain after breast cancer surgery is a major problem and women undergoing mastectomy and breast reconstruction experience post-operative pain syndromes in approximately one-half of all cases. Patients who have undergone breast reconstruction after mastectomy can suffer from acute postoperative pain with moderate or strong tension. In some cases, chronic neuropathic pain syndromes may occur after surgery. Opioids are used to treat pain, with serious side effects. The systemic postoperative analgesic regimen as thiocochlicoside P.O. along with paracetamol and NSAIDs I.V., which may limit the administration of opioids without reducing pain relief, seems to be necessary. Materials and Methods: This study was a clinical trial randomizing 70 patients undergoing breast reconstruction. Two main protocols of systematic post-operative analgesia, one using thiocochlicoside (group A) and the other without them (group B), were used. Both groups received paracetamol X3 and lornoxicam X2 I.V. systematically. The pain measurement scale (NPS) used to measure post-operative pain. Likert scales were used to evaluate patient's satisfaction and the difficulty from the side effects. An anonymous questionnaire was used for the data collection. Results: Statistically significant difference was found between pain on the day of surgery $(p=0.017)$ as well as the three subsequent days $(\mathrm{p}=0.000)$. In group $\mathrm{A}$, pain was reduced directly to half $\left(\mathrm{X}^{2}\right.$ surgery pain $=$ 93.888, $\mathrm{p}=0.000$ ) especially on the first post-operative day. In group A the satisfaction with analgesic treatment was higher than in group B $(\mathrm{p}=0.002)$. Conclusion: The use of thiocochlicoside in post-operative analgesia in breast reconstruction after mastectomy contributes to reduce the pain intensity experienced by patients and to reduce the side effects of opioid analgesics as a result of reduced demand for opioid analgesics. Patients who received the analgesia using muscle relaxants-spasmolytic reported greater satisfaction.
\end{abstract}

Keywords: Breast reconstruction- postoperative analgesia- multimodal analgesia- breast reconstruction pain

Asian Pac J Cancer Prev, 20 (3), 743-749

\section{Introduction}

All patients who have undergone breast reconstruction after mastectomy respond acute post-operative pain with moderate or strong intensity (Tasmuth et al.,1996; Wallace et al.,1996; Scott et al.,2011; American Cancer Society, 2013-2014). Its treatment has paramount importance as it reduces post-operative complications, facilitates recovery (Scott and Spittler, 2001; Barber et al., 2012; Ho Quoc and Delay, 2013; Kaya and Serel, 2013), reduces hospitalization days and reduces the likelihood of chronic pain's occurrence (Fassoulaki et al., 2005; Ballantyne, 2011).

It is common to use opioid analgesics for the treatment of post-operative pain (Papanastasiou and Evans, 2000; Pyati and Gan, 2005; Bonnet and Marret, 2007; Argoff and McCleane, 2009; Buvanendran and Kroin, 2009; Benzon et al., 2014; ASRA, 2016), with significant, often undesirable side effects and inadequate regard to safe use and monitoring of the patient.

Therefore, it is important to use an effective systematic post-operative analgesia regimen that will limit the administration of opioids without reducing pain relief.

The purpose of this study was to create a reliable and effective postoperative systematic analgesia protocol for patients undergoing breast reconstruction surgery using the application of a simple systematic post-operative analgesia regimen with muscle relaxant-spasmolytic (thiocochlicoside) drugs limiting the use of opioid analgesics.

${ }^{1}$ Department of Anesthesiology, Hellenic Anticancer Institute, ${ }^{3}$ Department of Plastic Surgery, Saint Savvas Hospital, ${ }^{2}$ Department of Medical Laboratories, ${ }^{4}$ Department of Nursing, University of West Attikis, ${ }^{5}$ Department of Nursing, Technological Educational Institute of Athens, Athens, Greece.*For Correspondence: mbourazani@yahoo.com 


\section{Materials and Methods}

Sample

The sample consisted of seventy patients with breast cancer, who had undergone mastectomy with immediate reconstruction with expanders, breast reconstruction after mastectomy with implants (replacement of expanders with silicone implants) or with autologous tissues (Lantissimus dorsi flap). All the participants gave their informed consent and the study conformed to the guidelines outlined in the Declaration of Helsinki and was approved by the Ethics Committees of the hospital.

The following criteria were used for the inclusion of the participants: Age between 20 and 65 years old, Ability to communicate in Greek, Diagnosis of breast cancer (BC), Cooperation and perceptual competence.

The exclusion criteria were as follow: patients who had BMI $>35$, patients who received treatment for chronic pain or/and any antipsychotic treatment, and patients who had Diabetes Mellitus.

\section{Methods}

Two main protocols of systematic post-operative analgesia were used: One group using muscle relaxants-spasmolytic, namely thiocochlicoside (brand name muscoril) constituted the group A, and another group using no additional muscoril constituted the group B. The two groups received paracetamol $1 \mathrm{gr}$ $\mathrm{x} 3$ and lornoxicam $8 \mathrm{mg}$ x2 intravenously. In addition, propoxyphene was administered intravenously to both groups only on demand, on severe acute pain, and only when the maximum permitted doses of simple analgesics had run out, in particular the increase of paracetamol to 1 gr $x 4$. Patients were divided into two groups by squared randomization sequence (35 patients per group).

A questionnaire, consisting of three parts, was used for the data collection. The first part included questions concerning the socio - demographic and clinical characteristics of the participants: age, weight and height, occupational status, place of residence and way of living. The clinical questions concerned the type of surgery, the medication for any concurrent illness, the additional treatment followed after the diagnosis of BC: hormone-therapy, chemotherapy or radiotherapy. The second part included questions of the Numbered Pair Scale (NPS) the general pain measuring tool on a scale from 0 to 10 , where 0 corresponded to no pain and 10 to the unbearable pain. The third part contained questions based on Likert scale in order to evaluate "the effectiveness of post-operative analgesia" as experienced by patients. One question related to "the satisfaction of systematic post-operative analgesia", and a second one related to the "difficulties from the side effects" of the analgesics. Patients were asked to choose between pre-replied answers from 1 to 5, where 1 corresponded to "not at all" and 5 to "very much".

The data collection took place between September 2016 and June 2017. The questionnaires were supplemented by a personal interview.

\section{Statistics}

The statistical analysis was based mainly in the Multivariate Statistical Analysis and more specifically in the Comparisons of Several Groups with regard to their individual characteristics. Frequency Tables and CrossClassification Tables were applied. Differentiation of mean values between groups was checked by applying nonparametric (Non-Parametric, Mann-Whitney and Wilcoxon Rank Sum test) tests if the normal or nonregularity assumptions of the Kolmogorov-Smirnov test were met. Differentiation techniques (ANOVA), or Kruskal-Wallis 1-way ANOVA techniques were applied for variations between the two groups depending on the satisfaction of the initial conditions required by the above techniques (normality Kolmogorov -Smirnov test, homosexuality Levin test.). Also for the pain variations per day and per group, the non-parametric check corresponding to the repeated measures ANOVA and in particular the Friedman test was applied.

A 95\% (Significance $\mathrm{p}<0.05$ ) Confidence Interval (C.I. $>95 \%$ ) considered to be particularly satisfactory for such measurements was considered to be a statistical significance level. For the statistical analysis the special statistical software SPSS v.24 was used, licensed by TEI of Athens.

\section{Results}

The post-operative pain of the breast reconstruction after mastectomy in both groups, on the day of surgery, reached $6 / 10$ of the numerical pain scale (NPS), with an average of 5.67/10 and a median of 6/10 (Table 1).

By separating the data by group of patients (Tables 2 and 3), it was found that from Day-1 onwards the two groups showed a statistically significant difference in pain intensity in the following days. Group A reported pain of $1 / 10$ intensity (median) at an average of 2.97 and median of 3 on the NPS, while group B reported pain of tension on the first post-operative day $5 / 10$ (prevailing value) with mean value of 4.8 and a median of 5 of the NPS. Group A on Day-2 experienced pain 2/10, with an average of 1.57 and a median of 2 of the NPS. Group B reported pain of $4 / 10$ intensity, with an average of 3.51 and a median of 4 of the NPS. On Day-3, group A reported no pain, that is,

Table 1. Post-Operative Pain after Breast Reconstruction Surgery in Both Groups

\begin{tabular}{lcccc}
\hline & $\begin{array}{c}\text { Day of surgery } \\
\text { DAY-0 }\end{array}$ & DAY-1 & DAY-2 & DAY-3 \\
\hline $\mathrm{N} \quad$ Valid & 70 & 70 & 70 & 70 \\
$\quad$ Missing & 0 & 0 & 0 & 0 \\
Mean & 5.67 & 3.89 & 2.54 & 1.76 \\
Median & 6.00 & 4.00 & 2.50 & 1.00 \\
Mode & 6 & 5 & 2 & 1 \\
Std. Deviation & 1.791 & 1.741 & 1.567 & 1.546 \\
Minimum & 0 & 1 & 0 & 0 \\
Maximum & 9 & 7 & 6 & 5 \\
\hline Statistics & & & &
\end{tabular}


The Role of Muscle Relaxants - Spasmolytic (Thiocochlicoside) in Postoperative Pain Management

Table 2. Frequency Distribution of Postoperative Pain after Breast Reconstruction Surgery in Group A

\begin{tabular}{lcccc}
\hline Group A & DAY-0 & DAY-1 & DAY-2 & DAY-3 \\
\hline $\mathrm{N} \quad$ & & & & \\
$\quad$ Valid & 35 & 35 & 35 & 35 \\
$\quad$ Missing & 0 & 0 & 0 & 0 \\
Mean & 5.14 & 2.97 & 1.57 & 0.71 \\
Median & 6.00 & 3.00 & 2.00 & 1.00 \\
Mode & 6 & $1 \mathrm{~b}$ & 2 & 0 \\
Std. Deviation & 1.958 & 1.465 & 1.065 & 0.789 \\
Minimum & 0 & 1 & 0 & 0 \\
Maximum & 8 & 5 & 3 & 3 \\
\hline
\end{tabular}

a Group A, With Thiococlicoside; b, Multiple modes exist; The smallest value is shown

Table 3. Frequency Distribution of Postoperative Pain after Breast Reconstruction Surgery in Group B

\begin{tabular}{lcccc}
\hline Group B & DAY-0 & DAY-1 & DAY-2 & DAY-3 \\
\hline $\mathrm{N}$ & & & & \\
$\quad$ Valid & 35 & 35 & 35 & 35 \\
$\quad$ Missing & 0 & 0 & 0 & 0 \\
Mean & 6.20 & 4.80 & 3.51 & 2.80 \\
Median & 6.00 & 5.00 & 4.00 & 3.00 \\
Mode & $6 \mathrm{~b}$ & 5 & 4 & 3 \\
Std. Deviation & 1.451 & 1.511 & 1.380 & 1.410 \\
Minimum & 2 & 1 & 0 & 0 \\
Maximum & 9 & 7 & 6 & 5 \\
\hline
\end{tabular}

b, Multiple modes exist; The smallest value is shown

the prevailing value was $0 / 10$, with an average of 0.71 and a median of 1 of the NPS, while group B reported pain in tension 3/10 (prevailing value), with an average of 2.8 and a median of 3 of the NPS.

Analyzing the data on patient satisfaction with post-operative analgesic treatment (Table 4), more than half $(51.4 \%)$ of the patients of group A reported "very" satisfied and $37.1 \%$ said they were "very much" satisfied. In group B the satisfaction of the patients was $17.1 \%$ and only $2.9 \%$ for those who declared "very" and "very much" respectively (Table 5). It is worth noting that in the group B 31.4\% declared "no" to "little" satisfaction with the analgesic treatment, fields that were not mentioned in the group A were used the thiocochlicoside.

Analyzing the data related to side effects, in group A (Table 6) 85.7\% reported having "no" difficulty with the

Table 4. Frequency Distribution of Satisfaction from Analgesic Treatment in Group A

\begin{tabular}{lcccc}
\hline Group A & Frequency & Percent & $\begin{array}{c}\text { Valid } \\
\text { Percent }\end{array}$ & $\begin{array}{c}\text { Cumulative } \\
\text { Percent }\end{array}$ \\
\hline Valid & & & & \\
Enough & 4 & 11.4 & 11.4 & 11.4 \\
Very & 18 & 51.4 & 51.4 & 62.9 \\
Very Much & 13 & 37.1 & 37.1 & 100.0 \\
Total & 35 & 100.0 & 100.0 & \\
\hline
\end{tabular}

Satisfaction From Analgesic Treatmenta
Table 5. Frequency Distribution of Satisfaction from Analgesic Treatment in Group B

\begin{tabular}{lcccc}
\hline Group B & Frequency & Percent & $\begin{array}{c}\text { Valid } \\
\text { Percent }\end{array}$ & $\begin{array}{c}\text { Cumulative } \\
\text { Percent }\end{array}$ \\
\hline Valid & & & & \\
$\quad$ Not At All & 2 & 5.7 & 5.7 & 5.7 \\
Little & 9 & 25.7 & 25.7 & 31.4 \\
Enough & 17 & 48.6 & 48.6 & 80.0 \\
Very & 6 & 17.1 & 17.1 & 97.1 \\
$\quad$ Very Much & 1 & 2.9 & 2.9 & 100.0 \\
$\quad$ Total & 35 & 100.0 & 100.0 & \\
\hline Satisfaction From Analgesic Treatment & &
\end{tabular}

Table 6. Frequency Distribution of Difficulty from Side Effects of Analgesic Treatment to Group A

\begin{tabular}{lcccc}
\hline Group A & Frequency & Percent & $\begin{array}{c}\text { Valid } \\
\text { Percent }\end{array}$ & $\begin{array}{c}\text { Cumulative } \\
\text { Percent }\end{array}$ \\
\hline Valid & & & & \\
No & 30 & 85.7 & 85.7 & 85.7 \\
Little & 4 & 11.4 & 11.4 & 97.1 \\
Very & 1 & 2.9 & 2.9 & 100.0 \\
Total & 35 & 100.0 & 100.0 & \\
\hline
\end{tabular}

Difficulty From The Side Effectecs

side effects, 11.4\% reported having "little" difficulty and finally $2.9 \%$ reported having "very" difficulty. Group B (Table 7) 60\% stated "no" difficulty with the side effects of drugs, $22.9 \%$ reported having "little" difficulty, 5.7\% reported having "enough", $8.6 \%$ reported having "very" and finally $2.9 \%$ reported having "Very much" difficulty. Having more difficulty with the side effects found in group B, and this may be attributed to the fact that Group B patients were more in pain, and were asking for additional analgesics when they were given an IV opioid, so the side effects reported were from the opioids.

\section{Statistics -Results}

The data check was used to verify their distribution and to decide the appropriateness of the statistical criteria to be applied. The variables were checked for regularity: (a) post-operation pain day-0, (b) post-operation pain day-1, (c) post-operation pain day-2, (d) post-operation pain day-3, (e) satisfaction from the post-operation pain management (f) Difficulties from the side-effects of the

Table 7. Frequency Distribution of Difficulty from Side Effects of Analgesic Treatment to Group B

\begin{tabular}{lcccc}
\hline Group B & Frequency & Percent & $\begin{array}{c}\text { Valid } \\
\text { Percent }\end{array}$ & $\begin{array}{c}\text { Cumulative } \\
\text { Percent }\end{array}$ \\
\hline Valid & & & & \\
No & 21 & 60.0 & 60.0 & 60.0 \\
Little & 8 & 22.9 & 22.9 & 82.9 \\
Enough & 2 & 5.7 & 5.7 & 88.6 \\
Very & 3 & 8.6 & 8.6 & 97.1 \\
Very Much & 1 & 2.9 & 2.9 & 100.0 \\
Total & 35 & 100.0 & 100.0 & \\
\hline Diffy
\end{tabular}

Difficulty From The Side Effectecs 
Table 8. Descriptive Measures of Pain Scale for the Group A

\begin{tabular}{lllll}
\hline Group A & DAY-0 & DAY-1 & DAY-2 & DAY-3 \\
\hline $\mathrm{N}$ & & & & \\
$\quad$ Valid & 35 & 35 & 35 & 35 \\
$\quad$ Missing & 0 & 0 & 0 & 0 \\
Mean & 5.14 & 2.97 & 1.57 & 0.71 \\
Median & 6.00 & 3.00 & 2.00 & 1.00 \\
Std. Deviation & 1.958 & 1.465 & 1.065 & 0.789 \\
Variance & 3.832 & 2.146 & 1.134 & 0.622 \\
Range & 8 & 4 & 3 & 3 \\
\hline Statistics & & & &
\end{tabular}

Statistics

Table 9. Descriptive Measures of Pain Scale for the Group B

\begin{tabular}{lllll}
\hline Group B & DAY-0 & DAY-1 & DAY-2 & DAY-3 \\
\hline $\mathrm{N}$ & & & & \\
$\quad$ Valid & 35 & 35 & 35 & 35 \\
$\quad$ Missing & 0 & 0 & 0 & 0 \\
Mean & 6.20 & 4.80 & 3.51 & 2.80 \\
Median & 6.00 & 5.00 & 4.00 & 3.00 \\
Std. Deviation & 1.451 & 1.511 & 1.380 & 1.410 \\
Variance & 2.106 & 2.282 & 1.904 & 1.988 \\
Range & 7 & 6 & 6 & 5 \\
\hline
\end{tabular}

Statistics

analgesic treatment. The assumption of regularity for all the above variables was violated $(p<0.05)$ and therefore non-parametric criteria were used to compare the average values. There was a statistically significant difference between pain on Day-0 until Day-3 between the two groups. The Mann-Whitney U criterion found that there was a statistically significant difference between pain on the Day-0 $(\mathrm{p}=0.017)$ as well as the three following days $(\mathrm{p}=0.000)$.

These findings are confirmed by the descriptive measures of the two groups (Tables 8 and 9). In both groups the pain decreased over time, however the mean and the median were much lower in group A and even on

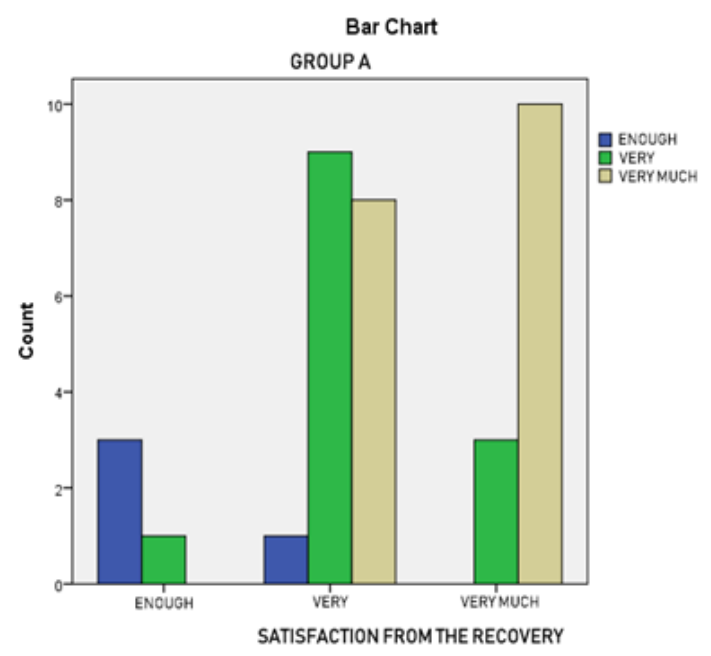

Figure 1. Comparative Chart of Analgesic Satisfaction and Improvement in Recovery for the Group A
Table 10. Quarterly of the Pain Scale for the Group A

\begin{tabular}{lcccc}
\hline & $\mathrm{N}$ & \multicolumn{3}{c}{ Percentiles } \\
& & $25^{\text {th }}$ & $50^{\text {th }}$ (Median) & $75^{\text {th }}$ \\
\hline Pain Day-0 & 35 & 4 & 6 & 6 \\
Pain Day-1 & 35 & 1 & 3 & 4 \\
Pain Day-2 & 35 & 1 & 2 & 2 \\
Pain Day-3 & 35 & 0 & 1 & 1 \\
\hline Descriptive Statistics & & & &
\end{tabular}

Table 11. Quarterly of the Pain Scale for the Group B

\begin{tabular}{lcccc}
\hline & $\mathrm{N}$ & \multicolumn{3}{c}{ Percentiles } \\
& & $25^{\text {th }}$ & $50^{\text {th }}($ Median $)$ & $75^{\text {th }}$ \\
\hline Pain Day-0 & 35 & 6 & 6 & 7 \\
Pain Day-1 & 35 & 4 & 5 & 6 \\
Pain Day-2 & 35 & 3 & 4 & 4 \\
Pain Day-3 & 35 & 2 & 3 & 4 \\
\hline
\end{tabular}

Descriptive Statistics $^{\mathrm{a}}$

the first postoperative day the pain was reduced directly to half of the pain reported in the day of the operation. The mean value from 5.14 on the NPS falls to 2.97 on the first day whereas the median of 6 was decreased to 3 . The corresponding values in the group receiving normal painkillers were the mean of 6.20 on the NPS falls to 4.80 on the first day while the median of $6 / 10$ was reduced to $5 / 10$.

Additional testing was used with non-parametric check corresponding to the repeated measures ANOVA and in particular the Friedman test in order to ascertain the statistically significant differences in pain between the days in each category separately. The findings were as follows: There was a statistically significant reduction in both groups receiving additional thiocochlicoside $\mathrm{X}^{2}(2)=93.888, \mathrm{p}=0.000$, and in the group of common pain relievers $X^{2}(2)=93.663, p=0.000$. However, reducing pain day by day in the group receiving extra thiocochlicoside was much greater. The tables 10 and 11, confirm ed the results of the Friedman test, as well as those mentioned above, for the median, which applied in

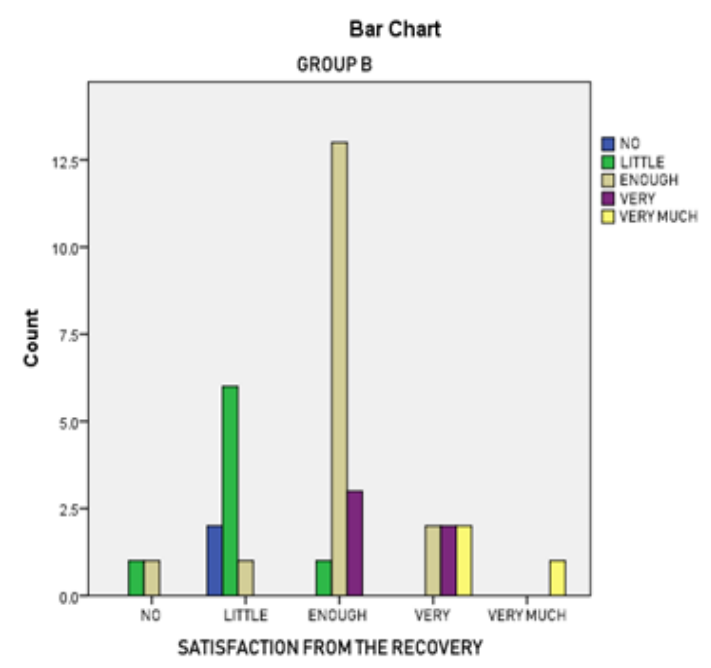

Figure 2. Comparative Chart of Analgesic Satisfaction and Improvement in Recovery for the Group B 
proportion to the other two quadrants of the NPS, which is $25 \%$ and $75 \%$. Note that by administering in addition to thiocochlicoside there was a $25 \%$ of women who feel no pain on the third day.

For the variables: (i) satisfaction from the post-operation pain management (f) Difficulties from the side-effects of the analgesic treatment both in the group A (Fisher's Exact Test, $p=0.002$ ) and in the group B (Fisher's Exact Test, $\mathrm{p}=0.000)$ there was a confirmed correlation (Figures 1 and 2). The level of analgesic satisfaction in group A starts from "quite a lot" and up to "very much", while in group B all the categories appeared. Finally, the variable "difficulties from the side effects of the analgesic treatment" was independent of the variable "satisfaction from the post-operation pain management".

Discussion of Results

The present study is original. There is no similar trial in the literature with the use of thiocochlicoside in post-operative analgesia for breast reconstruction after mastectomy.

The idea to use a spasmolytic-muscle relaxant emerged after investigation of the researchers. Observing the undesirable effects and the side-effects of opioid drugs - on the patients of the clinic - an attempt was made to find a multimodal post-operative analgesic regimen that would reduce their use.

Generally, as reported by many researchers (Fassoulaki et al., 2005; Hurley and Adams, 2011; Deyo and Ching, 2012) modern algorithm aims to use effective multimodal analgesic regimens, with analgesic protocols for each surgical procedure separately. Several studies highlighted the need for the reduction of opioid analgesics by postoperative analgesia (Correl et al., 2011; Burlacu et al., 2006; Wu and Raja, 2011), as their side effects delay the early mobilization and recovery of patients (Gottrup et al., 2000; Middleton, 2003; Katz et al., 2005; Dumestre et al., 2017). Several researchers have used multiple formulas with different categories of medicines (Afonso et al., 2017; Dumestre et al., 2017), such as anti-epileptic pregabalin and gabapentin (Fassoulaki et al., 2005, ; Chang et al., 2014; Eipe et al., 2015) which have been reduced due to increased costs and their side effects, especially dizziness, which used to make early mobilization difficult.

Then, based on Schneider's (2002) clinical studies who used methocarbamol, a centrally acting muscle relaxant as a supplement to the symptomatic treatment of musculoskeletal disorders associated with painful spasm, the idea came to carry out a trial using a corresponding drug available at the central pharmacy of the Athenian hospital, a drug that can be cheap and will not increase the cost of medical expenses and be relatively safe in use.

In the present study, postoperative pain experienced by patients in breast reconstruction surgery after mastectomy, on the day of surgery, reaches $6 / 10$ of the pain scale, for all patients, irrespective ly of the study group they belong to. In addition, in both groups pain intensity decreases over time. These findings are also confirmed by patient testimonies as recorded by other researchers (Wallace et al., 1996; Tasmuth et al., 1996; Stanley et al., 2012; Nesland and Steinbrook, 2013).

Separating data by group showed a statistically significant reduction in pain intensity, both in the group receiving additional muscle relaxant-spasmolytic $(p=0.000)$ and in the group of common pain killers $(p=0.000)$. However, the results revealed that the reduction in pain intensity per day was greater in the group receiving thiocochlicoside.

In particular, from the very first post-operative day, both mean and median values were much lower, with a statistically significant result $(\mathrm{p}=0.017)$, in patients receiving thiocochlicoside. It is also noteworthy that pain in group A was reduced directly to half the day of surgery (day-0). This finding can be inferred by the therapeutic properties of the muscle relaxant-spasmolytic agent used in the present study, thiocochlicoside, also used in the study of Umarkar et al., (2011). Their study mentioned the anti-inflammatory and analgesic effect of thiocochlicoside in post-traumatic and post-operative pain, as well as its use as a muscle relaxant. These findings also have similar outcomes to Schneider's (2002) results in clinical trials with methocarbamol.

Special emphasis was placed on the fact that on the second postoperative day, the majority ( $40 \%)$ of group A experienced pain 2/10 NPS, in contrast to group B who had $37 \%$ experienced pain 4 , double pain intensity. This is extremely important because following an Enhanced Recovery After Surgery (ERAS, 2017) program, patients could leave the hospital with a $2 / 10$ intensity pain that can be easily treated at home, offering psychosocial benefits to the patient and public- and be inexpensive for the health system. It is also important that $25 \%$ of the patients who received muscle relaxant-spasmolytic analgesic stated they had no pain on the third post-operative day, which also reinforces the abovementioned point of view, that patients can be discharged in the 2nd or, in some cases, on the 1 st postoperative day.

Therefore, the use of muscle relaxant-spasmolytic agents, in particular thiocochlicoside, may contribute to the reduction in pain intensity experienced by patients who have undergone breast reconstruction surgery after mastectomy.

In the present study, we also examined the patients' satisfaction with their analgesic treatment and any side effects from the analgesic drugs during their recovery.

As the data revealed satisfaction with analgesic treatment is undoubtedly related to improved recovery for both groups ( $p=0.000, p=0.002)$. But what is remarkable here is that satisfaction in group A who received additional thiocochlicoside started from the category "enough" to "very much" on the LIKERT scale, unlike the other group where all the categories were answered, namely " no "to" very much ", with a significant percentage of them (31.4\%) declaring" no "to" little "satisfaction. In short, Group A patients were more satisfied with the analgesic medication they received.

From the analysis of the data on the side effects experienced by patients from their analgesic treatment, someone can easily understand the difference between the two groups. Group A patients mostly reported (85.7\%) "no" difficulty with side effects of drugs, respectively group B patients reported "no" difficulty in a lower percentage $(60 \%)$. This finding is similar to that of Hidalco 
and Pusic (2005) who used methocarbamol in their study, in women with breast reconstruction. Also, a larger range of responses to group B admitted more difficulties from the side effects. This phenomenon is attributed to the fact that group B patients, because they were in more pain, were asking for an extra painkiller, so they were given opioid (propoxyphene) intravenously. Therefore, the side effects reported by the patients concerned the side effects from opioids: dizziness, nausea and/or vomiting (Buvanendran and Kroin, 2009; Gritsenko et al., 2014).

Finally, the present study investigated the participants' view of improving their recovery from their analgesic treatment. The results of the study revealed that patients who received additional muscle relaxant-spasmolytic analgesics in their treatment reported that their recovery was improved from "enough" to "very much". On the other hand, patients in group B reported, respectively, improvement from "no", which means that patients who received additional thiocochlicoside experienced greater improvement in their recovery.

In conclusion, the present study introduced the way for the use of thiocochlicoside as muscle relaxant-spasmolytic drug in post-operative analgesia, while restricting the required opioid drugs and consequently their side effects. However, it would be important for the study to be repeated in other centers or in a larger number of patients.

\section{Conclusions and Suggestions}

The efficacy of postoperative analgesia should be evaluated and recorded on the patient's chart on a regular basis so that it can be revised when it is inadequate.

The use of muscle relaxant-spasmolytic agents (thiocochlicoside) in postoperative analgesia in breast reconstruction procedures after mastectomy helps to reduce the pain intensity experienced by patients and to reduce the side effects of opioid analgesics as a result of a reduced demand for opioid analgesics. Patients who received the form of systematic post-operative analgesia using thiocochlicoside drugs reported greater satisfaction with their analgesic treatment and significant improvement in their recovery.

The main suggestion of this work is to encourage the use of muscle relaxant-spasmolytic drugs in breast reconstruction procedures after mastectomy, in the context of a postoperative systemic analgesia protocol. This is an inexpensive and effective method of multifactorial postoperative analgesia. It presents very good results for the patient by limiting the use of opioids postoperatively, without the occurrence of complications affecting the recovery of patients.

\section{Conflict of interests}

The authors declare no conflict of interests.

\section{References}

Afonso AM, Newman MI, Seeley N et al (2017). Multimodal analgesia in breast surgical procedures: Technical and Pharamacological considerations for Liposomal Bupivacaine use. Plast Reconstr Surg, 5, 1480-1.

American Cancer Society (2013-2014). Breast Cancer treatment,
Breast Cancer Facts and Figures; p 23-26, Available at: \{https://www.cancer.org/content/dam/cancer-org/research/ cancer-facts-and-statistics/breast-cancer-facts-and-figures/ breast-cancer-facts-and-figures-2013-2014.pdf $\}$. Accessed at: 20/12/2016.

American Society of Regional Anesthesia and Pain Medicine [ASRA] (2016). Implementing Therapy with Opioids in Cancer Patients - American Society of Regional Anesthesia and Pain Medicine. Available online: \{http://www.asra. com/pain-resource/article/31/implementing-therapy-withopioids-in-can . Accessed at 30/8/2017.

Argoff CE, McCleane G (2009). Pain management secrets. 3rd CDN Philadelphia. PA: Mosby Elsevier.

Ballantyne J (2011) Chronic pain after surgery or injury. International Association for the Study of Pain (IASP). 19(1). Available online: \{http://www.iasppain.org/ PublicationsNews $\}$. Accessed at: 17/9/2017.

Barber M, Thomas J, Dixon J (2012). Breast cancer: Visual Guide for Clinicians. Oxford: Clinical Pub. Available online: $\{\mathrm{http}: / / \mathrm{www} . c$ linicalpublishing.co.uk\}. Accessed at: $11 / 12 / 2017$.

Benzon H, Raja S, Fishman S, Liu S, Cohen S (2014). Essentials of Pain Medicine. St. Louis: Elsevier Health Sciences.

Bonnet F, Marret E (2007). Postoperative pain management and outcome after surgery. Best Pract Res Clin Anaesthesiol, 21, 99-107.

Burlacu CL, Frizelle HP, Moriarty DC, Buggy DJ (2006). Fentanyl and clonidine as adjunctive analgesics with levobupivacaine in paravertebral analgesia for breast surgery. Anaesthesia, 61, 932-7.

Buvanendran A, Kroin JS (2009). Multimodal analgesia for controlling acute postoperative pain. Curr Opin Anaesthesiol, 22, 588-93.

Chang CY, Challa CK, Shah J, Eloy JD (2014). Gabapentin in acute postoperative pain management. Biomed Res Int. 2014:631756. Available online: \{https://www.ncbi.nlm.nih. gov/pmc/articles/PMC4009126/\}. Accessed at: 23/08/2017.

Correll DJ, Viscusi ER, Grunwald Z (2001). Epidural analgesia compared with intravenous Morphine PatientControlled Analgesia: Postoperative outcome measures after mastectomy with immediate TRAM Flap breast reconstruction. Reg Anesth Pain Med, 26, 445-9.

Deyo R, Ching A (2012). Surgical trials for pain relief: In search of better answers. Pain, 153, 2155-6.

Dumestre DO, Webb CE, Temple-Oberle C (2017). Improved recovery experience achieved for women undergoing implant-based breast reconstruction using an enhanced recovery after surgery model. Plast Reconstr Surg, 139, 550-9.

Eipe N, Penning J, Yazdi F, et al (2015). Perioperative use of pregabalin for acute pain-a systematic review and meta-analysis. Pain, 156, 1284-1300.

Enhanced Recovery after Surgery ERAS (2017). ERAS SOCIETY. Available online: \{http://erassociety.org/ guidelines/list-of-guidelines $\}$. Accessed at: 06/11/2017.

Fassoulaki A, Triga A, Melemeni A (2005). A Multimodal analgesia with gabapentin and local anesthetics prevents acute and chronic pain after breast surgery for cancer. Anesth Analg, 101, 1427-32.

Gritsenko K, Khelemsky Y, Kaye AD, Vadivelu N, Urman RD (2014). Multimodal therapy in perioperative analgesia. Best Pract Res Clin Anaesthesiol, 28, 59-79.

Gottrup H, Andersen J, Arendt-Nielsen L, Jensen TS (2000). Psychophysical examination in patients with post-mastectomy pain. Pain, 87, 275-84.

Hidalgo DA, Pusic AL (2005). The role of methocarbamol and intercostal nerve blocks for pain management in breast 

augmentation. Aesthet Surg J, 25, 571-5.

Ho Quoc C, Delay E (2013). Breast reconstruction after mastectomy, Journal de Gynecologie. J Gynecol Obstet Biol Reprod, 42, 29-39.

Hurley RW, Adams MCB (2011). Perioperative pain management. In: Miller RD, Pardo MC. Basics of anesthesia. Edited by Elsevier Saunders, Philadelphia 6th edition, pp 650-62.

Katz J, Poleshuck EL, Andrus CH, Hogan LA, Jung BF (2005). Risk factors for acute pain and its persistence following breast cancer surgery. Pain, 119, 16-25.

Kaya B, Serel S (2013). Breast reconstruction. Exp Oncol, 35, 280-6. Available online: \{https://www.ncbi.nlm.nih.gov/ pubmed/24382438 \}. Accessed at: 20/12/2017.

Middleton C (2003). Understanding the psychological effects of unrelieved pain. Nurs Times, 99, 28-9.

Nesland JM, Steinbrook RA(2013). A retrospective cohort study of post mastectomy pain syndrome. The Scientific World Journal, (Article ID 159732). Available online: \{http:// dx.doi.org/10.1155/2013/159732\}. Accessed at 23/08/2017.

Papanastasiou S, Evans J (2000). Postoperative analgesia in augmentation mammoplasty. Plast Reconstr Surg, 105, 1241.

Pyati S, Gan TJ (2005). Periopertive pain management. CNS Drugs, 21, 185-211.

Schneider MS (2002). Methocarbamol: adjunct therapy for pain management in breast augmentation. Aesthet Surg $J$, 22, 380-1.

Scott L, Christopher J (2001). Breast reconstruction with implants and expanders. Plast Reconstr Surg, 107, 177-87.

Spear SL, Spittler CJ (2001). Breast reconstruction with implants and expanders. Plast Reconstr Surg, 107, 177-87.

Stanley SS, Hoppe IC, Ciminello FS (2011). Pain control following breast augmentation: a quality systematic review. Aesthet Surg J, 32, 964-72.

Tasmuth T, Von Smitten K, Kalso E (1996). Pain and other symptoms during the first year after radical and conservative surgery for breast cancer. Br J Cancer, 74, 2024-31.

Umarkar AR, Bavaskar SR, Yewale PN (2011). Thiocochlicoside as muscle relaxant: a review. Int J Pharm Biol Sci, 1, 364-71.

Wallace MS, Wallace AM, Lee J, Dobke MK (1996). Pain after breast surgery: a survey of 282 women. Pain, 66, 195-205.

Wu CL, Raja SN (2011). Treatment of acute postoperative pain. Lancet, 377, 2215-25.

\section{c) (i) \&}

This work is licensed under a Creative Commons AttributionNon Commercial 4.0 International License. 